\title{
Políticas de información en las universidades públicas estatales 1990-1999 en el contexto del Consejo Nacional para Asuntos Bibliotecarios
}

\author{
Egbert Sánchez Vanderkast *
}

Artículo recibido: 28 de noviembre de 2008.

Artículo aceptado:

21 de enero de 2009.

\section{RESUMEN}

El objetivo de este estudio es presentar las acciones efectuadas por el Consejo Nacional para Asuntos Bibliotecarios de 1990 a1999, para establecer políticas de información en las universidades públicas estatales. Y también mostrar cómo estas acciones y estrategias influyeron en particular para formular el Plan Nacional de Desarrollo Bibliotecario y una política sobre desarrollo institucional de las colecciones bibliográficas.

Palabras clave: Asociaciones de Bibliotecas; Políticas de información; Desarrollo de Colecciones; Planificación de Bibliotecas.

* Centro Universitario de Investigaciones Bibliotecológicas de la UNAM, México. egbert@servidor.unam.mx

INVESTIGACIÓN BIBLIOTECOLÓGICA, Vol. 23, Núm. 47, enero/abril, 2009, México, ISSN:
0187-358X. pp. 67-89 
Information policies in public state Mexican universities, 1990-1999 within the context of the National Council for Library Matters

Egbert Sánchez Vanderkast

The purpose of this study is to present those actions undertaken between 1990 and 1999 by the National Council for Library Matters, in order to establish information policies in public state Mexican universities. And to find out whether these strategies had any particular effect in the formulation of a National Library Development Plan and the establishment of an institutional bibliographic collection development policy.

Keywords: Library Associations, Information policies; Collection development; Library planning.

\section{INTRODUCCIÓN}

L as asociaciones por lo general tienen la función de promover el desarrollo profesional, potenciar el trabajo de los profesionales, asegurar una representación adecuada ante las administraciones públicas, y ofrecerles servicios a sus miembros.

En el caso específico de las bibliotecas y las unidades de información se podría añadir a las funciones mencionadas la de promover la cooperación y el desarrollo de las instituciones que están a cargo de los bibliotecólogos y profesionistas afines.

La Asociación de Bibliotecarios de Instituciones de Enseñanza Superior e Investigación (ABIESI), fundada en 1957, y el Consejo Nacional para Asuntos Bibliotecarios de las Universidades Públicas Estatales, CONPAB-UPES (1990) son agrupaciones reconocidas en el contexto de la educación superior e impulsoras de políticas de información ${ }^{1}$ en este nivel educativo.

Posteriormente a mediados de los noventa se dejó de hablar de la ABIESI, en tanto que el CONPAB-UPES adquirió un gran impulso debido principal-

1 La temática "políticas de información” ha sido tratada con amplitud por autores anglo e hispano parlantes como: Almada Navarro (1999), Cornella (1998), Fernández Bajón (2001), Gómez Fernández-Cabrera (1999), Morales Campos (con una vasta producción sobre la temática, la más recién publicada en 2003), Páez Urdaneta (1990), Ros García y López Yepes (1994) entre otros. 
mente al ímpetu de sus agremiados, los responsables de los sistemas bibliotecarios de las universidades públicas estatales.

Desde 1984 este Consejo ha seguido reuniéndose en distintos estados de la República Mexicana.

El CONPAB, desde su creación en 1990, ha realizado y sigue realizando varias acciones para promover el desarrollo de los sistemas bibliotecarios de las UPES y ha puesto también énfasis en la formulación de una política para el desarrollo de colecciones bibliográficas.

Las acciones del CONPAB durante el periodo 1990-1999 definitivamente no podrían ser analizadas sin considerar el contexto de la planeación de la educación superior, ni el propio entorno de los sistemas bibliotecarios.

\section{CONTEXTO DE PLANEACIÓN DE LA EDUCACIÓN SUPERIOR}

La cultura de la planeación de las bibliotecas universitarias está estrechamente ligada a la propia planeación de las instituciones de educación superior (IES) debido a que tales aspectos repercuten de forma directa e indirecta en toda la estructura de los sistemas bibliotecarios universitarios.

Respecto a lo anterior Del Valle precisa:

la planeación forma parte desde hace tiempo de las prácticas institucionales $\mathrm{y}$ [contribuyen] al proceso de toma de decisiones $\mathrm{y}$, en general, al funcionamiento institucional. $^{2}$

Como es sabido, el desarrollo de la educación superior de 1970 a 1990 enfrentaba variados retos: la crisis financiera y cualitativa de la educación; la evaluación y la asignación presupuestal, y la concentración en los interlocutores y protagonista de la educación superior, lo cual llevó a las IES a tener poco éxito en la aplicación de políticas innovadoras que pudieran transformar a la organización estructuralmente hablando.

Por esta razón se podrían apreciar dos visiones del mismo fenómeno, una al interior de las IES y la otra la que existe entre el gobierno federal y las IES.

Pallan Figueroa ${ }^{3}$ al realizar un balance en 1993 sobre la planeación en el desarrollo de la educación superior enunció algunas de las posibles causas de

2 J. Hanel del Valle. (1993), "Panorámica de la planeación de la educación superior en México", en Fernández, A. L. y Santini L., Dos décadas de la planeación de la educación superior, Mexico, D. F., ANUIES, p. 15.

3 C. Pallan Figueroa, (1993), "La planeación en la historia del desarrollo universitario", en Fernández, A. L. y Santini L., Dos décadas de la planeación de la educación superior, Mexico, D. F., ANUIES, pp. 35-52. 
los resultados poco exitosos, las que se pueden resumir en lo siguiente:

- Los condicionantes de la situación educativa; las fallas en cuanto a concepción y estrategias; la naturaleza de las técnicas utilizadas; las actitudes de los responsables de la planeación (la posición tecnocrática ingenua versus la creación original), y las deficiencias en el plan operativo en el que se destacan otros problemas, como eran:

- Medios insuficientes de financiamiento.

- Participación insuficiente y falta de interés entre los miembros de las IES.

- Falta de continuidad en las acciones emprendidas por cada institución.

El desarrollo logrado fue haber integrado los cuadros de planeación (la creación de unidades técnicas de planeación o de planes de desarrollo) y el haber implantado el Sistema Nacional para la Planeación Permanente de la Educación Superior (SINAPPES).

Se tenía la esperanza de que con la identificación de los protagonistas de la Planeación de la Educación Superior (PES): los actores técnicos, los grupos directivos, los grupos técnicos, las comunidades universitarias y los sistemas colegiados de planeación institucional y los protagonistas externos, se auguraba el éxito de los resultados de los planes de desarrollo.

Hacia los años noventa la planeación normativa ${ }^{4}$ inició una transición hacia el dinamismo de la planificación estratégica y algunos indicios de ésta fueron:

a) La realización de un proceso de diagnóstico centrado más en la detección de problemas de la educación superior que en descripciones.

b) El interés —en el plano formal declarativo — de impulsar un cambio cualitativo en la situación de la educación superior.

c) La promulgación de políticas dirigidas a lograr propósitos predefinidos.

d) La apertura de la participación en función de la cual han tenido lugar la implementación de formas colegiadas de participación en la planeación.

e) La instalación de sistemas y redes de información para la toma de decisiones. 5

4 El concepto técnico tiene que ver con el deber ser regulador, véase A. L. Fernández, "Evaluación de la planeación de la educación superior”, en Fernández, A. L. y Santini L., (1993), Dos décadas de la planeación de la educación superior, Mexico, D. F., ANUIES, p. 29. 
Estos sistemas y redes de información se inclinaban hacia los datos más para la toma de decisiones, que para obtener información científico técnico y acceso a información.

Fernández confirma lo anterior al manifestar que "los sistemas y redes de información se han orientado más al asunto tecnológico (hardware) que al de la accesibilidad y oportunidad de la información". ${ }^{6}$

En el contexto de la planeación de la educación superior, Llarena de Thierry $^{7}$ al analizar el impacto de la planeación en el desarrollo de este tipo de educación destaca:

- La formación y actualización de los profesores.

- El fomento de la planeación al interior de las IES.

- La atención a la demanda de educación superior.

- La reforma de algunos aspectos de la educación superior.

Sin embargo Thierry no atendía de manera particular los servicios bibliotecarios y de información como parte del desarrollo de la capacidad para buscar y procesar información que permitiera acceder a nuevos conocimientos, lo que sin lugar a dudas podría haber repercutido en la alta calidad de las IES.

\section{CONTEXTO DE LOS SISTEMAS BibliotecARIOS}

El contexto de los sistemas bibliotecarios universitarios debía ser analizado a partir del programa marco PRONAES, Programa Nacional de Educación Superior.

Según Salvador Malo, Director General de Investigación Científica y Superación Académica de la SEP, una de las acciones importantes de dicho programa tendría que ser el reforzamiento de los sistemas bibliotecarios. Esto confirmaba que las IES proponían el mejoramiento de las bibliotecas universitarias del país en general pero incluyendo el mejoramiento de los acervos; los sistemas de procesamiento técnico; y las instalaciones de cada biblioteca.

Para ello la SEP convocó en 1984 a los responsables de las bibliotecas de las universidades públicas estatales para analizar las posibilidades, las experiencias y las necesidades de cada una de ellas, y establecer acciones que llevaron al mejoramiento de las bibliotecas en general.

$6 \quad$ Ibidem.

7 R. Llarena de Thierry, (1990), "Impacto de la planeación en el desarrollo de la educación superior”, en Fernández, A. L. y Santini L., Dos décadas de la planeación de la educación superior, México, D. F., ANUIES, pp. 53-84. 
Cuadro 1: Antecedentes de temas tratados en las

Reuniones de Responsables de los Sistemas Bibliotecarios de las Universidades Públicas Estatales

\begin{tabular}{|c|c|c|c|}
\hline Reunión & Ciudad Sede & Año & Temas \\
\hline 1 & Colima, Col. & 1984 & $\begin{array}{l}\text { A. Capacitación y actualización de recursos humanos. } \\
\text { B. Apoyos interinstitucionales para el mejoramiento de los ser- } \\
\text { vicios bibliotecarios. } \\
\text { C. Función de las autoridades universitarias en el mejoramiento } \\
\text { de los servicios bibliotecarios. } \\
\text { D. Instalaciones y equipo de las bibliotecas universitarias. }\end{array}$ \\
\hline 2 & $\begin{array}{l}\text { Campeche, } \\
\text { Camp. }\end{array}$ & 1985 & $\begin{array}{l}\text { A. Desarrollo y evaluación de colecciones. } \\
\text { B. Programa de formación de recursos humanos. } \\
\text { C. Programa de cooperación. }\end{array}$ \\
\hline 3 & $\begin{array}{l}\text { Cuernavaca, } \\
\text { Mor. }\end{array}$ & 1986 & $\begin{array}{l}\text { A. Evaluación de la Primera Etapa de los Cursos de Adiestra- } \\
\text { miento para Personal no Profesional de los Sistemas Biblio- } \\
\text { tecarios de las Universidades Públicas Estatales, UPES. } \\
\text { B. Planteamiento y examen de propuestas relativas a la necesi- } \\
\text { dad de crear cursos abiertos de bibliotecología a nivel técnico } \\
\text { y/o licenciatura y /o especialización. }\end{array}$ \\
\hline 4 & $\begin{array}{l}\text { Villahermosa, } \\
\text { Tab. }\end{array}$ & 1987 & $\begin{array}{l}\text { Redes de cooperación bibliotecaria: evaluación, integración, } \\
\text { funcionamiento. }\end{array}$ \\
\hline 5 & $\begin{array}{l}\text { Tampico, } \\
\text { Tamp. }\end{array}$ & 1988 & $\begin{array}{l}\text { A. Selección y adquisición de materiales. } \\
\text { B. Capacitación. } \\
\text { C. Metodología para la evaluación de bibliotecas. } \\
\text { D. Automatización y redes bibliotecarias. } \\
\text { E. Cooperación bibliotecaria. }\end{array}$ \\
\hline 6 & $\begin{array}{l}\text { Guadalajara, } \\
\text { Jal. }\end{array}$ & 1989 & $\begin{array}{l}\text { Elaboración del Programa Nacional de Desarrollo Bibliotecario de } \\
\text { las Universidades Públicas Estatales. Dicho programa abarcaba } \\
\text { cinco grandes temas. } \\
\text { 1. Capacitación y adiestramiento del personal bibliotecario. } \\
\text { 2. Desarrollo y actualización de acervos. } \\
\text { 3. Redes de cooperación. } \\
\text { 4. Modernización de los servicios bibliotecarios con el apoyo de } \\
\text { la automatización. } \\
\text { 5. Cooperación internacional basada en el "Mexican Project } \\
\text { Center". }\end{array}$ \\
\hline
\end{tabular}

8 Sin embargo 1998 fue la excepción, debido que el proyecto de solicitud de apoyo financiero para realizar la Reunión en Saltillo (21 al 23 de octubre de 1998) carecía de aprobación por parte de la Dirección General de Educación Superior y la Dirección de Desarrollo Universitario de la Secretaría de Educación Superior (SEP). En ese año 2008 los responsables de los Sistemas Bibliotecarios de las Instituciones de Educación Superior se reunieron en Puebla, Puebla. 


\begin{tabular}{|c|c|c|l|}
\hline Extraordinaria & Colima, Col. & 1989 & Red Nacional de Bibliotecas. \\
\hline 7 & $\begin{array}{c}\text { Ciudad Obre- } \\
\text { gón, Son. }\end{array}$ & 1990 & $\begin{array}{l}\text { Visualización y análisis de las estrategias del Programa Nacional } \\
\text { de Desarrollo Bibliotecario de las Universidades Públicas Estata- } \\
\text { les. }\end{array}$ \\
\hline
\end{tabular}

Elaborado por el autor, 2008.

En síntesis las Reuniones de Responsables de los Sistemas Bibliotecarios fueron un laboratorio para desarrollar trabajos en equipos multidisciplinarios con un mismo objetivo, el de mejorar los servicios bibliotecarios en todos sus aspectos. Las mesas de trabajo, proporcionaron un mejor panorama de las situaciones problemáticas por las que estaban atravesando los diferentes sistemas bibliotecarios de las universidades estatales.

Por otro lado, la integración y consolidación como grupo no ha sido una tarea fácil por los siguientes factores:

a) La heterogeneidad de sus miembros, lo que se veía inicialmente como un impedimento.

b) Los frecuentes cambios que se daban a nivel institucional con la rotación de funcionarios en la coordinación, dirección o jefatura de las bibliotecas.

Naturalmente estos factores influyeron y retrasaron la concreción de acciones para establecer políticas de información en las bibliotecas o unidades de información de educación superior del país.

\section{Consejo PARA ASUnTOS Bibliotecarios De LAS UNIVERSIDADES PÚBLICAS ESTATALES (CONPAB-UPES)}

El CONPAB-UPES tenía sus antecedentes en la Comisión Nacional de Representantes de Responsables de Universidades Públicas Estatales.

El objetivo de esta Comisión era presentar los avances de los últimos cinco años y que las acciones que esperaban los representantes fueran consideradas por la SEP.

De estas acciones se presentaron como programa:

- La adquisición, actualización e incremento de los acervos bibliohemerográfico.

- Los Cursos de capacitación, actualización y adiestramiento.

- El aprovechamiento del desarrollo tecnológico con el fin de automatizar los 
servicios y promover la creación de la Red Nacional de Bibliotecas de las Instituciones de Educación Superior (RENABIES) del sector público. ${ }^{9}$

Esto podría traducirse en políticas de información sobre:

- Desarrollo de Colecciones.

- Educación Bibliotecológica.

- Infraestructura.

Lo cual se podría interpretar como parte de la planeación estratégica que obtendrían las bibliotecas y los servicios bibliotecarios.

Con la propuesta de organización del Consejo para Asuntos Bibliotecarios de las Universidades Públicas Estatales (CONPAB-UPES), en 1990, se da el primer paso para establecer el CONPAB-UPES. ${ }^{10}$

La justificación que antecede a esta acción fue impulsada por el Programa para la Modernización Educativa 1989-1994 y el documento Red Nacional de Bibliotecas (RENABIES) propuesto por la ANUIES en la XXIII Asamblea General celebrada en Cuernavaca, Morelos, que ofrecieron las condiciones para que los Sistemas Bibliotecarios de las Universidades Públicas Estatales contaran

con una agrupación que [tuvieran] representatividad nacional ante las autoridades competentes, así como ante organismos públicos y privados nacionales e internacionales. ${ }^{11}$

Una de las labores inmediatas que realizó el CONPAB-UPES fue darle continuidad y seguimiento a las conclusiones y recomendaciones de las mesas de trabajos y los temas tratados durante la Reunión de Guadalajara. ${ }^{12}$

9 Reunión de responsables de los sistemas bibliotecarios de las universidades Públicas estatales, (7, 1990, Cd. Obregón, Sonora), p.104.

10 Los objetivos que en primera instancia persiguió este Consejo fueron: 1.Propiciar el desarrollo de los servicios bibliotecarios de las UPES; 2 . Fomentar el intercambio de experiencias entre los responsables de los sistemas bibliotecarios de las UPES; 3. Identificar e implementar soluciones comunes a la problemática de los Sistemas Bibliotecarios de las UPES; 4. Promover la cooperación bibliotecaria a partir de acciones coordinadas en redes regionales; 5 . Definir concretar y organizar acciones tendientes a crear la Red Nacional de Bibliotecas de las UPES", véase Reunión de responsables de los sistemas bibliotecarios de las universidades públicas estatales, (7: 1990, Cd. Obregón, Sonora), pp. 73-74.

11 Reunión de responsables de los sistemas bibliotecarios de las universidades Públicas estatales, (7, 1990, Cd. Obregón, Sonora), p. 73.

12 Los temas tratados durante la Reunión de Guadalajara fueron: 1. "Capacitación y adiestramiento del personal bibliotecario; 2. Desarrollo y actualización de acervos; 3. Redes de cooperación; 4. Modernización de los servicios bibliotecarios con apoyo de la automatización; 5. Cooperación internacional partiendo del "Mexican Project Center", véase, Reunión de responsables de los sistemas bibliotecarios de las universidades públicas estatales, (7, 1990, Cd. Obregón, Sonora), p. 106. 
Por consiguiente en la reunión de Ciudad Obregón (1990) se retomaron las temáticas de: Redes de cooperación; Capacitación; Automatización; y Acervos (desarrollo de acervos), y las conclusiones que emanaron de la mesa de trabajo:

- Redes de cooperación, el CONPAB sugirió crear una comisión para atender estas conclusiones.

- Capacitación; una de las acciones inmediata fue cambiar el Programa de Capacitación a Programa de Formación de Recursos Humanos.

- Automatización, se propuso crear la comisión para la automatización.

- Acervos (desarrollo de acervos).

Las sugerencias aprobadas fueron:

A. Crear una Comisión Especial para que se avoque a establecer los lineamientos científicos, generales y específicos que propicien el desarrollo de las colecciones bibliográficas de las UPES.

B. Realizar un diagnóstico integral de todos los recursos de los servicios de información disponibles con el objeto de establecer acciones concretas y facilitar la toma de decisiones.

C. El presupuesto mínimo dedicado a las colecciones bibliográficas debería ser del 2\% al 5\% indicado por las normas de ABIESI y debería ser aplicado exclusivamente para la adquisición de recursos documentales.

D. El financiamiento proveniente de la SEP para los servicios bibliotecarios debería estar sujeto al cumplimiento de los siguientes requisitos:

1. Que exista un Plan de Desarrollo Bibliotecario Institucional.

2. Que exista una Politica para el Desarrollo de las colecciones bibliográficas.

La frecuencia de aparición de las temáticas mencionadas revela que las situaciones problemáticas que giraban alrededor de ellas no habían sido resueltas en su totalidad, o las soluciones propuestas habrían sido sólo de carácter paliativo.

La integración de comisiones sugeridas por los responsables de CONPAB fue una de las primeras iniciativas de este Consejo, para atacar desde la raíz el problema de los acervos y buscar llegar al fondo de la problemática.

No obstante había una normatividad "las Normas para el Servicio Bibliotecario de la ABIESI" aceptada por la ANUIES en 1968, pero ésta había sido letra muerta. 
Por lo tanto se buscaba tener un Diagnóstico Integral para realizar una planeación estratégica basada en lineamientos científicos que impulsara de manera cuantitativa el desarrollo de colecciones.

En este caso un diagnóstico ayudaría a evidenciar las fortalezas y las debilidades del acervo bibliográfico, y presentaría las oportunidades y las amenazas que repercutían en los servicios bibliotecarios.

\section{COMISIÓN DIAGNÓSTICO INTEGRAL DEL ESTADO ACTUAL DE LOS SISTEMAS BIBLIOTECARIOS DE LAS UPES}

En 1990, el CONPAB-UPES solamente contaba con el esbozo de la situación general de las Universidades Públicas Estatales (UPES) de 1984, donde cada responsable de su sistema bibliotecario de una manera global daba a conocer, las características y los principales problemas que enfrentaba su sistema bibliotecario o de bibliotecas.

En 1992 en la Reunión de los Responsables celebrada en Puebla se integró una Comisión Especial para realizar el Diagnóstico Integral del Estado Actual de los Sistemas Bibliotecario de las UPES, cuyos integrantes fueron los responsables de los sistemas bibliotecarios de las universidades de Yucatán, Guanajuato, Baja California e Hidalgo y la Red de los Sistemas de Bibliotecas de las Universidades del Centro, A.C. (RESBUIC). El objetivo de esta Comisión Especial fue

Avocarse a localizar y reunir la información disponible sobre el programa de evaluación de la educación superior con el fin de comunicarla posteriormente a éstos. $^{13}$

La justificación que se encontraba detrás del interés de hacer este diagnóstico se debió en primera instancia a la carencia de información sobre cómo operaría el programa de evaluación de la Comisión Nacional para la Evaluación de la Educación Superior (CONAEVA), y en segunda instancia al desconocimiento de la forma para elaborar y presentar propuestas de apoyo para el Programa Fondo para la Modernización de la Educación (FOMES).

13 E. Ortiz Marín y Piña Marquina, J. I. (1994), Modelo para evaluar bibliotecas universitarias mexicanas, aplicación en los sistemas bibliotecarios de cinco universidades públicas estatales, Guanajuato, Gto., los autores, (Tesis de Maestría, Universidad de Guanajuato). p. 4. 
Durante 1992, la Subsecretaría de Educación Superior e Investigación Científica (SESIC) ${ }^{14}$ invitó a los miembros del grupo de trabajo del CONPAB sobre Diagnóstico Integral del Estado Actual de los Sistemas Bibliotecario de las UPES a sumar esfuerzos y apoyarse mutuamente en el desarrollo del proyecto "Estudios de indicadores básicos de los servicios bibliotecarios de las universidades", contemplado en el convenio SESIC-ANUIES para el desarrollo universitario que formaba parte del Programa 5. Fomento a los Servicios Bibliotecarios e Informativos.

Por consiguiente requerían de datos actualizados de los sistemas bibliotecarios de las UPES.

El alcance de esta acción permitió tener una visión general de cada uno de los sistemas bibliotecarios de las Universidades Públicas Estatales (UPE). Sin embargo, no exponía las contribuciones de los miembros del CONPAB, ni el intercambio de experiencias que se dio entre ellos, los cuales quedaron plasmados en los acuerdos, las recomendaciones y los compromisos que fueron adquiridos al concluir las reuniones nacionales de los Representantes de los Sistemas Bibliotecarios de las UPES.

\section{ACUERDOS, RECOMENDACIONES Y COMPROMISOS ADQUIRIDOS}

\section{DURANTE LAS OCHO REUNIONES DE LOS RESPONSABLES DE LOS SISTEMAS BIBLIOTECARIOS DE LAS UNIVERSIDADES PÚBLICAS ESTATALES, (UPES)}

En 1992, el segundo año de la instalación de Consejo para Asunto Bibliotecarios de las Universidades Públicas Estatales (CONPAB-UPES), los responsables hicieron un alto en el camino para realizar una evaluación de los acuerdos, recomendaciones y compromisos adquiridos durante las ocho reuniones nacionales, de 1984 a 1991.

En la reunión de Colima (1984) se tomaron 4 acuerdos; en Campeche (1985): 7; Cuernavaca (1986): 2; Villahermosa (1987): 4; Tampico, 6; Guadalajara (1989): 6; Ciudad Obregón (1990): 8; y Xalapa (1991): 14.

Esta evaluación fue encomendada a Ignacio Piña Marquina.

14 La invitación a los miembros del grupo de trabajo del CONPAB por parte de la SESIC fue con el fin de que apoyáramos a ésta a desarrollar uno de los proyectos (3.3. Estudios de indicadores básicos de los servicios bibliotecarios de las universidades) contemplado en el convenio SESICANUIES para el desarrollo universitario Programa 5. Fomento a los servicios bibliotecarios e informativos, tal y como lo indica el título del proyecto se trata de levantar un inventario de las bibliotecas de las UPES,(véase Ortiz Marín, E. y Piña Marquina, J. I., Modelo para evaluar bibliotecas universitarias mexicanas, aplicación en los sistemas bibliotecarios de cinco universidades públicas estatales, Guanajuato, Gto., los autores, (Tesis de Maestría, Universidad de Guanajuato), p. 122. 
Los acuerdos tomados en las mencionadas reuniones dejaron precedente de la visión de los responsables de los sistemas bibliotecarios respecto a las temáticas ya mencionadas.

Casi un $32.25 \%$ de los 93 acuerdos y recomendaciones formulados durante el periodo 1984-1990 presentados por Piña Marquina en la IX Reunión celebrada en la ciudad de Puebla y sintetizados en el documento Acuerdos, recomendaciones y compromisos adquiridos durante las ocho Reuniones de los Responsables de los Sistemas Bibliotecarios de las Universidades Públicas Estatales, (UPES) fueron cumplidos.

Esto indica que no todos los acuerdos pueden ser resueltos de inmediato.

Todo dependía del contexto ${ }^{15}$ que aglutinaba estas recomendaciones, y en ocasiones algunas requerían de plazos más amplios y de mayores recursos económicos para darles solución.

Muchos de los acuerdos de las reuniones de responsables fueron integrados de manera sistemática a las recomendaciones de las temáticas del Programa de Red Nacional de Bibliotecas de las Instituciones de Educación Superior (RENABIES) del sector público, la cual fue aprobada en la XXIII Reunión Ordinaria de la Asamblea General de la ANUIES celebrada en Cuernavaca, Morelos, (1990): Diseño y Reglamentación de la Red o Normatividad; Homologación y Automatización; Capacitación y Adiestramiento; y Desarrollo y Actualización de Acervos todos que a continuación se desglosa:

15 El contexto a principio de los años noventa estuvo determinado por: El Programa para la Modernización Educativa 1989-1994, a nivel federal; El sector de las Instituciones de Educación Superior por medio de la ANUIES con el documento Consolidación y Desarrollo del Sistema Nacional de Educación Superior y La propuesta de los responsables de los Sistemas Bibliotecarios (en la actualidad CONPAB) para aprovechar del desarrollo tecnológico con el fin de automatizar los servicios y promover la creación de la Red Nacional de Bibliotecas de las Instituciones de Educación Superior (RENABIES) del sector público. Véase Documento base aprobado en el XXIII Reunión Ordinaria de la Asamblea General de la ANUIES. Cuernavaca, Morelos, 1990. Red Nacional de Bibliotecas, "Con este programa se busca mejorar, diversificar y ampliar los servicios bibliotecarios que ofrecen las instituciones de educación superior y crear una red de bibliotecas que se apoye en las tecnologías modernas de comunicación", (véase Revista de la Educación Superior, (1990), Vol. 19 núm. 1 (73) enero-marzo, p.81). 


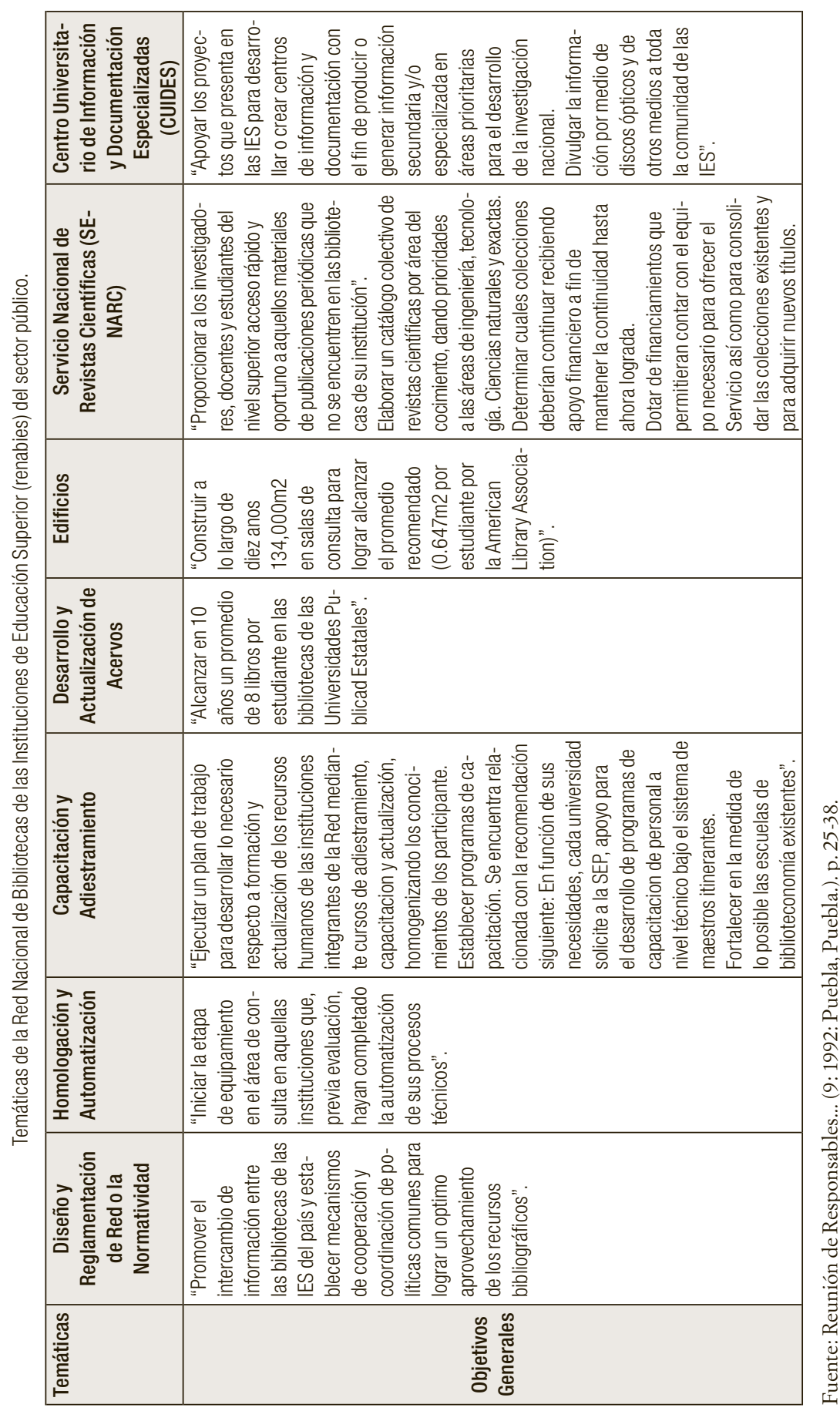


Todas estas recomendaciones hechas por la RENABIES tienen sustento en los acuerdos tomados en las Reuniones de Responsables de los Sistemas Bibliotecarios y en la visión de los Responsables que asistieron a dichas reuniones.

Los resultados del Diagnóstico Integral del Estado Actual de los Sistemas Bibliotecarios de las UPES, las recomendaciones recopiladas y presentadas en la IX Reunión, celebrada en la ciudad de Puebla, en el documento Acuerdos, recomendaciones y compromisos adquiridos durante las ocho Reuniones de los Responsables de los Sistemas Bibliotecarios de las Universidades Públicas Estatales, (UPES) por Ignacio Piña Marquina; la normatividad existente en relación a los procesos técnicos y servicios expuestos en el Capítulo 4. De la Administración, Estructura y Normatividad, artículo 19 relativo a la Red Teleinformática; artículo 20 relativo a la Descripción Bibliográfíca; artículo 21 relativo al Intercambio de la información, y el Capítulo 5 De los Servicios, artículos 23 al 25 del Reglamento de la Red Nacional de Bibliotecas de las Instituciones de Educación Superior (RENABIES) y las Normas para el Servicio Bibliotecario de Enseñanza Superior y de Investigación de la ABIESI (1968) sirvieron para identificar, analizar y discutir los siguientes temas:

1. Desarrollo de Recursos Humanos.

2. Desarrollo de Colecciones.

3. Organización Documental (procesos técnicos).

4. Desarrollo y Consolidación de Servicios.

5. Cooperación Bibliotecaria Interinstitucional (redes).

6. Fortalecimiento de la Infraestructura, (edificios, mobiliario, equipos, cómputo, telecomunicaciones, audiovisual).

De esa forma los temas mencionados se convirtieron en los ejes de acción prioritarios del Plan Nacional de Desarrollo Bibliotecario de las Universidades Públicas Estatales, 1994-2000, el objetivo general del cual, decía, es:

promover el desarrollo de los sistemas bibliotecarios de las Universidades Públicas Estatales (UPES) a partir de estrategias que propicien la obtención de apoyos por parte de los organismos vinculados con la Educación Superior (ES) y la Investigación Científica (IC), para que cuenten con las condiciones que les permitan responder con servicios de excelencias a las demandas de las comunidades académicas insertas en las dinámicas de la competitividad nacional e internacional. ${ }^{16}$ (11, 1994, Chihuahua. Chih.). 
Para reforzar este objetivo general al tratar el tema de Financiamiento y Apoyos Adicionales de SEP a las Universidades en la Reunión de Guanajuato (1993) el encargado de la Dirección General de Investigación Científica y Superación Académica, ya había hecho una exhortación a los coordinadores, directores y jefes de los sistemas bibliotecarios de las UPES para: "que la institución dedique recursos propios para las bibliotecas, como una cantidad fija anual, ya que lo que otorga la SEP son realmente apoyos adicionales". ${ }^{17}$

Por consiguiente la Comisión de Elaboración y Redacción del Plan Nacional de Desarrollo Bibliotecario de las Universidades Públicas Estatales 19942000 hacía la siguiente recomendación:

para hacer viable y operativo este plan es recomendable que las instancias de autoridad de cada Universidad Pública Estatal (UPE) y las autoridades federales establezcan compromisos para dar vigencia a las Normas para el Servicio Bibliotecario de la ABIESI, adoptadas por la ANUIES en su Reunión Anual celebrada en Xalapa, Ver., en abril de $1968 .^{18}$

Esos ejes de acción prioritarios podrían ser considerados como programas de información del Plan Nacional de Desarrollo Bibliotecario de las Universidades Públicas Estatales, que con el transcurrir del tiempo se convertían en políticas de información institucional.

Al presentar el Plan Nacional de Desarrollo Bibliotecario de las Universidades Públicas Estatales al Director General de Educación Superior de la SEP en marzo de 1996, él puso especial énfasis en:

- Los Recursos Humanos, y encomendó un proyecto de Formación Profesional en Biblioteconomía a la Dirección de la Escuela Nacional de Biblioteconomía y Archivonomía (ENBA), que tendría la responsabilidad de elaborar y plantear una propuesta para su análisis en la próxima Reunión del CONPAB (1997), porque esta temática había sido una constante en las discusiones de las mesas de trabajo durante las reuniones celebradas desde 1984 hasta 1996.

- Las Redes Regionales, y puntualizó la necesidad de promover y propiciar su fortalecimiento, que por consiguiente permitiría optimizar información sobre los servicios bibliotecarios y de información; y los recursos de información en general.

El tema de redes fue tratado principalmente en la Reunión de Tampico de 1988 y figuró en la agenda como cooperación en diversas mesas

17 Ibíd, h.13.

18 Ibid. 
de trabajo tanto de las Reuniones de los Responsables de los Sistemas Bibliotecarios como del CONPAB.

- El Desarrollo de Colecciones; sugirió que las instancias de autoridad tomaran conciencia de asignar y mantener un porcentaje presupuestal inamovible para esta actividad.

En varias ocasiones los Responsables de los Sistemas Bibliotecarios habían apelado al buen juicio de las autoridades invitándolos a apegarse a las Normas para el Servicio Bibliotecario de la ABIESI.

- La Construcción de Parámetros de Evaluación; le encargó al CONPAB que asumiera el compromiso de construir los parámetros de evaluación que servirían para realizar el análisis de la realidad bibliotecaria de cada UPE con el fin de definir las normas mínimas que impulsaran a los sistemas bibliotecarios a un desarrollo armónico y planeado.

Los puntos tratados por el Director General de Educación Superior de la SEP tuvieron los siguientes resultados:

\section{Recursos humanos}

El CONPAB se había adelantado a las recomendaciones del Director General de Educación Superior de la SEP, Víctor Arredondo ya que en la Reunión celebrada en Durango (1995) la propuesta había sido

Delinear los parámetros del modelo de biblioteca universitaria que requiere el proyecto de modernización educativa a partir del diagnóstico de cada uno de los Sistemas Bibliotecarios de las UPES y de las propuestas que en materia de desarrollo bibliotecario, plantean los programas nacionales de educación superior, asî como desahogar los puntos de interés para los miembro de CONPAB-UPES, ${ }^{19}$

Esto se discutió en las Mesas de Trabajo "la Propuesta de las Licenciaturas en Biblioteconomía y Archivonomía en la Modalidad Abierta y a Distancia de la ENBA", que iniciaban sus actividades académicas en 1997. (12, 1995, Durango, Dgo.), p. 3. 


\section{REDES REGIONALES}

El Consejo Nacional para Asuntos Bibliotecarios de las UPES (CONPAB-UPES) incluyó de manera oficial a las Redes Regionales en las Reuniones Nacionales de los Responsables de los Sistemas Bibliotecarios de las UPES, como Reunión CONPAB - RENABIES, por lo que se celebraron tres reuniones de 1996 a 1999. Después de 1999 ya no se menciona a la RENABIES.

Los logros, avances y proyectos de cada una fueron presentados siguiendo la siguiente estructura:

A. La Situación que guarda cada Red Regional en su Desarrollo.

B. El Desarrollo de Planes de Trabajo.

Debido a las características propias y necesidades diferentes de cada Red: Para alcanzar las metas propuestas son indispensables los recursos económicos necesarios por lo que al informar sobre las actividades de la RENABIES, Porfirio Támez Solís (Representante de la región Noreste) comentó

los recursos financieros en nuestras instituciones han sido y tal vez por mucho tiempo lo serán insuficientes para atender todas las necesidades que ellas enfrentan, sin embargo, creemos que es urgente que las Instituciones Públicas de Educación Superior asignen presupuestos propios y suficientes para la operación y desarrollo de sus bibliotecas. ${ }^{20}$

Por lo antes expuesto este Programa de RENABIES no logró alcanzar las expectativas de éxito de los Sistemas Bibliotecarios. Después de 1999 se dejó de hablar del RENABIES.

\section{Desarrollo de colecciones}

En el marco de la RENABIES se promovió el Programa Consorcio Mexicano de Bibliotecas de Investigación (COMBI) cuyo objetivo era

ofrecer servicios de información que apoyen de manera eficiente y oportuna las actividades de docencia e investigación a través de la conexión a bases de datos internacionales vía Internet. ${ }^{21}$

20 Reunión de Responsables de los Sistemas Bibliotecarios de las Universidades Públicas Estatales, (13, 1996, San Luis Potosí, SLP), p. 79.

21 Reunión de Responsables...(12, 1995, Durango, Dgo.), p. 71. 
El costo de este servicio de recuperación y suministro de documentos fue absorbido por la SEP en su primer año de existencia. Mientras que la capacitación corrió por cuenta de la ANUIES. Este proyecto fue tema de discusión durante las Reuniones Nacionales de los Responsables de los Sistemas Bibliotecarios de las UPES de Durango (1995) y de la primera reunión CONPABRENABIES celebrado en San Luis Potosí (1996).

El Programa Consorcio Mexicano de Bibliotecas de Investigación (COMBI) se transformó en el Sistema de Información Documental, pero paulatinamente se dejó de mencionar este programa.

\section{LA CONSTRUCCIÓN DE PARÁMETROS DE EVALUACIÓN}

En la Reunión efectuada tanto en Mexicali como en Tijuana (1997) se presentó el Segundo Diagnóstico de los Servicios Bibliotecarios de las Universidades Públicas Estatales de México, el cual se publicó bajo el título Situación de los Servicios Bibliotecarios de las Universidades Públicas Estatales de México ${ }^{22}$ en 1997 con la siguiente estructura: 1. Datos Generales; 2. Estructura Organizacional; 3. Recursos Humanos; 4. Recursos Documentales; 5. Servicios; 6. Recursos Materiales (edificios, equipos y presupuesto); 7. Automatización; y 8. Cooperación.

Este Diagnóstico contribuyó a contar con datos actualizados a fin de integrar la propuesta de Modelo para la Evaluación Integral de las Bibliotecas de Educación Superior en México.

Este modelo contemplaba los indicadores de los aspectos que componen el servicio bibliotecario (acceso a los recursos documentales, número y tipo de los servicios ofrecidos, estantería, formación de usuarios de la información, días y horas de servicios); los insumos (recursos documentales, recursos humanos, recursos financieros, recursos materiales, estructura organizacional), y los procesos (inventario y evaluación de la colección documental, políticas de desarrollo de la colección, adquisición de materiales documentales, y catalogación y clasificación).

Cada indicador tenía un parámetro asignado, y éste a su vez incluía las medidas asignadas para la obtención de los datos. Este proyecto se concluyó pero no se aplicó esta forma de evaluación debido a que la atención estaba dirigida hacia la certificación ISO 9000.

22 Véase A. Arellano Rodríguez y Verdugo Sánchez, J. A., (2000), Situación de los Servicios Bibliotecarios de las Universidades Públicas Estatales de México, México, D.F., SESIC/DGES-SEP, ENBA. 
La Reunión del CONPAB celebrada en Saltillo (1999) le dio seguimiento a los proyectos que eran prioritarios para las UPES:

- La generación de indicadores de evaluación.

- La actualización del diagnóstico.

- La formación y capacitación de recursos humanos.

- La formalización del Catálogo Bibliográfico Colectivo.

- La formación de Usuarios.

Se le dio seguimiento a la propuesta de Modelo para la Evaluación Integral de las Bibliotecas de Educación Superior en México.

Otro punto importante que fue tratado en esta Reunión de los Sistemas Bibliotecarios de las UPES fue cómo obtener recursos adicionales o sacar provecho de los programas instituidos por la SEP.

Como ejemplo se encuentran los siguientes casos:

- Para la formación de los recursos humanos en bibliotecología:

que en los proyectos del Programa de Apoyo al Desarrollo Universitario (PROADU) se dé preferencia a las solicitudes relacionadas con la profesionalización del personal bibliotecario a fin de desarrollar recursos humanos capaces de satisfacer la demanda de los servicios de información... ${ }^{23}$

- En cuanto a los Proyectos Bibliotecarios se dijo:

Que en el caso de que por política institucional los apoyos a los Sistemas bibliotecarios, sean solicitados a través de las DES (Dependencias de Educación Superior)[,] que cada Universidad haya definido los proyectos, [que]se hagan en coordinación con el Responsable del Sistema Bibliotecario, tanto para asegurar la continuidad de las colecciones hemerográficas, como para considerar todos los aspectos relativos al incremento y puesta en servicio de los acervos. ${ }^{24}$

- En cuanto al Desarrollo de Colecciones se acordó:

Que se establezca con claridad que los recursos documentales que se autoricen vía PROMEP (Programa de Mejoramiento del Profesorado), de la

23 Reunión de Responsables de los Sistemas Bibliotecarios de las Universidades Públicas Estatales, (15, 1999, Saltillo, Coahuila), p. 70.

24 Ibid., p. 69. 
institución, y que se encuentran bajo la custodia de los académicos, pero que requieren ser inventariados y registrados por el Sistema Bibliotecario, a donde deben regresar una vez que el académico termine su trabajo, o se separe de la institución. ${ }^{25}$

y también que los proyectos que fueron financiados por el programa Fondo para la Modernización de la Educación Superior (FOMES)

Que se establezca con claridad que los recursos documentales que se autoricen vía FOMES desde cada una de las DES (Dependencias de Educación Superior), no salen del ámbito de competencia del sistema bibliotecario, en donde deberán inventariarse, registrarse y ponerse en servicio de acuerdo a las políticas establecidas, incluso, que es conveniente y necesario el trabajo coordinado desde la selección y adquisición de los acervos para optimizar los presupuestos asignados. ${ }^{26}$

Es visible el apoyo institucional que recibió cada uno de los proyectos.

\section{Consideraciones finales}

Los responsables de los sistemas bibliotecarios han capitalizado las experiencias, las propuestas y los acuerdos tomados en las mesas de trabajo en un programa que tenía tres ejes temáticos: 1 . acervos; 2. la formación de recursos humanos; y 3. la creación de la Red Nacional de Bibliotecas de las Instituciones de Educación Superior, RENABIES.

La constitución de los responsables de los sistemas bibliotecarios, en el Consejo Nacional para Asuntos Bibliotecarios de las Universidades Públicas Estatales, CONPAB-UPES, refleja la madurez de ellos como grupo de trabajo. A la vez externaron el deseo de que existiera un Plan de Desarrollo Bibliotecario Institucional, así como también una Política de Desarrollo de Colecciones Bibliográficas.

Esto los llevó a buscar los mecanismos y las estrategias siguientes:

- Instituir una comisión de diagnóstico sobre el estado actual de los sistemas bibliotecarios de las UPES.

- Evaluar y cumplir los acuerdos, recomendaciones y compromisos adquiridos por los responsables de los Sistemas Bibliotecarios de las Universidades de 1984 a 1991. 
- Revisar y realizar propuestas al reglamento de la red Nacional de Bibliotecas de las Instituciones de Educación Superior, RENABIES.

Todo lo anterior coadyuvó a identificar los ejes temáticos que deberían ser considerados en un Plan Nacional de Desarrollo Bibliotecario para las UPES.

Quedó de manifiesto el impulso del CONPAB para formular una política de desarrollo de colecciones que se hizo visible en:

- La exhortación que hizo a las autoridades universitarias a fin de apegarse a las Normas para el Servicio Bibliotecario de la ABIESI.

- La promoción que le dio al programa Consorcio Mexicano de Bibliotecas de Investigación (COMBI).

- La construcción de parámetros de evaluación por medio de la propuesta de un "Modelo para la Evaluación Integral de las Bibliotecas de Educación Superior".

- Los proyectos específicos que buscaban asegurar la continuidad de las colecciones; la puntualización del destino final de las colecciones adquiridas por programas ya establecidas como FOMES, Fondo para la Modernización de la Educación Superior, PROMEP, Programa de Mejoramiento del Profesorado y PROADU, Programa de Apoyo al Desarrollo Universitario.

Sin lugar a dudas, durante el periodo de 1990 a 1999 el CONPAB-UPES fungió como catalizador, actor, gestor y promotor en el proceso de implantación de políticas de información, implícitas o explícitas, en el marco de las Universidades Públicas Estatales de México.

\section{OBRAS CONSUltadAs}

Almada de Ascencio, M. (1999), "Política de información y politicas educativas: un vínculo importante para el desarrollo hemisférico”, en Boletín de política informática, Vol. 22 (6) pp. 34-43.

Arellano Rodríguez, A. y Verdugo Sánchez, J. A., (2000), Situación de los Servicios Bibliotecarios de las Universidades Públicas Estatales de México, México, D.F., SESIC/DGES-SEP, ENBA.

Cornella, A. (1998), "Information policies in Spain”, en Government Information Quarterly. Vol. 15 (2) p. 197-220. 
Fernández, A. L. (1993), "Evaluación de la planeación de la educación superior”, en Fernández, A. L. y Santini L., Dos décadas de la planeación de la educación superior. México, D. F.: ANUIES.

Fernández Bajón, M. T. (2001), Políticas de información y documentación en la España del siglo XIX, Gijón, Trea.

Gómez Fernández-Cabrera, J., (1999), "Políticas de información y documentación”, en García Gutiérrez, Introducción a la documentación informativa y periodística, Alcalá de Guadaira, Sevilla, Editorial MAD.

Hanel del Valle, J. (1993), "Panorámica de la planeación de la educación superior en México”, en Fernández, A. L. y Santini L. Dos décadas de la planeación de la educación superior, México, D. F., ANUIES, 1993. p. 15.

Lopez Yepes; J. (2004), Diccionario enciclopédico de ciencias de la documentación, Madrid, Editorial Síntesis.

Llarena de Thierry, R. (1993), "Impacto de la planeación en el desarrollo de la educación superior”, en Fernández, A. L. y Santini L., Dos décadas de la planeación de la educación superior, México, D. F., ANUIES.

México, Poder Ejecutivo Federal, (1989), Programa para la Modernización Educativa, 1989-1994, México, Talleres de encuadernación progreso, 1989.

Morales Campos, E. (2003), Infodiversidad, globalización y derecho a la información, Buenos Aires, Sociedad de Investigaciones Bibliotecológicas.

Ortiz Marín, E. y Piña Marquina, J. I. (1994), Modelo para evaluar bibliotecas universitarias mexicanas, aplicación en los sistemas bibliotecarios de cinco universidades públicas estatales, Guanajuato, Gto., los autores, (tesis de Maestría, Universidad de Guanajuato).

Pallan Figueroa C. (1993), "La planeación en la historia del desarrollo universitario”, en Fernández, A. L. y Santini L. Dos décadas de la planeación de la educación superior, México, D. F., ANUIES.

Páez Urdaneta, I. (1990), Información para el progreso de América Latina, Caracas, Congreso de la República, Universidad Simón Bolívar.

Reunión Anual del Consejo Nacional para Asuntos Bibliotecarios de las Instituciones de Educación Superior, (9, 1992, Puebla, Pue.), (1992), Memorias del noveno Encuentro Nacional de Responsables de Sistemas Bibliotecarios de Universidades Públicas y Estatales, Puebla , Pue., Universidad Autónoma de Puebla.

Reunión Anual del Consejo Nacional para Asuntos Bibliotecarios de las Instituciones de Educación Superior, (11, 1994, Chihuahua, Chih.), (1994), Memorias y documentos de trabajo de la onceava reunión nacional de responsables de sistemas bibliotecarios de las Universidades Públicas Estatales, Chibuahua, Chih., Universidad Autónoma de Chihuahua. 
Reunión Anual del Consejo Nacional para Asuntos Bibliotecarios de las Instituciones de Educación Superior, (12, 1995, Victoria de Durango, México), (1996), Memoria y documentos de trabajo de la duodécima Reunión de Responsables de los Sistemas Bibliotecarios de las Universitarias Públicas Estatales, México, D. F., Universidad Juárez del Estado de Durango, CONPAB/UPES, 1996, 93 p.

Reunión Anual del Consejo Nacional para Asuntos Bibliotecarios de las Instituciones de Educación Superior, (13, 1996, San Luis Potosí, S.L.P.), (1996), Memorias y documentos de trabajo de la Decimotercera Reunión Nacional de Responsables de los Sistemas Bibliotecarios de las Universidades Públicas Estatales, CONPAB/UPES y primera reunión CONPAB/RENABIES, 1996 , San Luis Potosí, S. L. P., San Luis Potosí, S.L.P., Universidad Autónoma de San Luis Potosí.

Reunión de Responsables de los Sistemas Bibliotecarios de las Universitarias Públicas Estatales, (7, 1990, Cd. Obregón, Sonora), (1990), Memoria de la $7^{a}$ Reunión de Responsables de Bibliotecas Universitarias Públicas Estatales, del 7 al 9 de marzo de 1990, Cd. Obregón, Sonora, Durango, Dgo., Consejo para Asuntos Bibliotecarios de las Universidades Públicas Estatales; SEP, Dirección General de Investigación Científica y Superación Académica, Universidad Juárez del Estado de Durango.

Reunión de Responsables de los Sistemas Bibliotecarios de las Universidades Públicas Estatales, (15, 1999, Saltillo, Coah.), (2000), Memorias y documentos de trabajo de la xv Reunión del Consejo $\mathrm{Na}$ cional para Asuntos Bibliotecarios de las Universidades Públicas Estatales y III Reunión CONPAB RENABIES, Saltillo, Coahuila, Universidad Autónoma de Coahuila, coordinación General del Sistema de Infotecas Centrales.

Rodríguez Gómez, R. (1999), “The modernization of higher education”, en Higher Education Policy (1999), vol. 12 (1) pp. 53-67.

Ros García, J., López Yepes, J., (1994), Políticas de información y documentación, Madrid, Editorial Síntesis.

Rowlands, I. (1996), "Understanding information policy”, en Journal of information science, Vol. 22 (1)p p.13-25

Rubin, R. E. (1998), Foundations of library and information science, New York, Neal-Schuman Publisher. 